\section{Mindfulness, cancer, and pain}

\author{
Anna M. Tacón \\ Department of Health, Exercise and Sport \\ Sciences, Texas Tech University, Lubbock, \\ TX, USA
}

\section{Abstract}

Pain is the most distressing and incapacitating symptom experienced by cancer patients. While mindfulness-based stress reduction (MBSR) interventions have been conducted with cancer patients, no study has explored psychological and pain-related factors in patients with cancer-related pain. The effects of an eight-week MBSR intervention were investigated on pre-post scores for pain catastrophizing, pain-related anxiety, pain intensity ratings, and mental adjustment to cancer in breast cancer patients with pain. Forty-one women diagnosed with cancer-related pain participated in the study. The MBSR was conducted for 1.5 hours/week for eight weeks; participants were trained in the body scan, sitting meditation, and hatha yoga with formal guidance and direction as to daily homework practice. Results showed significant reduction in scores for pain catastrophizing, pain anxiety, and pain ratings; also significant positive changes occurred for mental adjustment to cancer. This study is the first to investigate MBSR effects for psychological factors in patients with cancer pain. Much more research is needed in this area.

\section{Introduction}

Pain is the most distressing, persistent, and incapacitating symptom experienced by cancer patients. ${ }^{1}$ Melzak and Wall's seminal Gate Control Theory of Pain ${ }^{2}$ explains pain as a multidimensional experience influenced by psychological factors, which strongly can affect a patient's pain adaptation . Cancer pain is complex, and may involve compressive, inflammatory, ischemic, and neuropathic mechanisms occurring at many regions. ${ }^{3}$ While biological and neuropathic pain etiologies are common in cancer, cognitive and affective aspects of cancer pain can more strongly contribute to pain ratings than sensory aspects. ${ }^{4}$ Psychological factors such as a patient's pain castrophizing, ${ }^{5}$ mental adjustment to cancer, ${ }^{6}$ and pain anxiety ${ }^{7}$ may affect the subjective pain experience. The purpose here is to explore the effects of a mindfulness-based intervention on pain-related psychological factors in breast cancer patients.

\section{Pain catastrophizing}

Pain catastrophizing (PC) is a cognitiveaffective pattern characterized by a preoccupation with pain and appraisals about the meaning of pain experiences. ${ }^{5}$ Catastrophizing plays a central role in the fear-avoidance model of chronic pain, for it is viewed as the cognitive route through which fear of pain develops, and accounts for $7-31 \%$ of the variance in pain severity; 8 thus, addressing the cognitive distortions enhanced by catastrophizing, such as through a cognitive-based intervention, may interrupt the chronic fear-avoidance cycle. Also, catastrophizing is associated with increased negative affect and pain intensity in those with cancer. ${ }^{9}$ No mindfulness study has investigated this factor in patients with chronic cancer pain.

\section{Pain anxiety}

Pain-related anxiety is a central construct of fear-avoidance models. Specifically, fearanxiety-avoidance models of chronic pain emphasize pain-related anxiety as a key diathesis in both the development and maintenance of chronic pain. ${ }^{7}$ Also, fear-anxietyavoidance models of chronic involve patterns similar to anxiety disorder models to where anxiety disorders either follow from, or are exacerbated by unpleasant experiences associated with avoidance, catastrophic cognitions, and major distress. ${ }^{10}$ Thus, similar processes may be involved in the development and maintenance of both chronic pain and anxiety disorder. Surprisingly, again, no mindfulness study has examined pain anxiety in a cancer population.

\section{Mental adjustment to cancer}

Mental adjustment to cancer refers to an individual's psychological and behavioral coping responses to having cancer.6,11 The implicit assumption is that coping will reflect an enduring attitudinal or behavioral style in individuals confronted with this disease. Five mental and behavioral coping strategies have been identified: Fighting Spirit (FS), where the patient accepts the diagnosis with an optimistic attitude; Helpless-Hopelessness ( $\mathrm{HH})$, refers to being overwhelmed by the diagnosis; Anxious Preoccupation (AP), is characterized by an anxious preoccupation with the disease; Stoic-Fatalism (FA), reflects a resigned attitude and passive acceptance about cancer; and, Avoidance (Denial), is where patients avoid or deny the notion of having cancer.6,11 One study with cancer patients demonstrated positive shifts in cancer adjustment after completing the MBSR intervention, 12 yet no study has explored intervention effects in patients suffering specifically with cancer pain.
Correspondence: Anna M. Tacón, Department of Health, Exercise and Sport Sciences, Texas Tech University, Mailstop 3011, Lubbock, TX 79409, USA. Tel. +806.790.5162 - Fax: +806.742.2342.

E-mail: anna.tacon@ttu.edu

Key words: mindfulness, cancer-related pain, pain catastrophizing, pain anxiety.

Received for publication: 28 September 2011 Revision received: 30 October 2011. Accepted for publication: 30 October 2011.

This work is licensed under a Creative Commons Attribution NonCommercial 3.0 License (CC BYNC 3.0).

@C Copyright A.M. Tacón, 2011

Licensee PAGEPress, Italy

Alternative Medicine Studies 2011; 1:e15

doi:10.4081/ams.2011.e15

\section{Mindfulness meditation}

In Kabat-Zinn's secular eight-week Mindfulness-Based Stress Reduction (MBSR) program, mindfulness is the disciplined practice of paying attention to the present moment with uncritical, non-judging acceptance. ${ }^{13}$ This discipline fosters detached, nonjudgmental observation or witnessing of thoughts, perceptions, sensations, and emotions, providing a means of self-regulating one's arousal and awareness. More specifically, mindfulness is operationally defined as a process of regulating attention in order to bring a quality of nonelaborative awareness to current experience and a quality of relating to one's experience within an orientation of curiosity, experiential openness, and acceptance. 14 The MBSR has been shown to significantly reduce stress levels and symptoms, scores for anxiety and depression, cancer distress, and fatigue in cancer patients.15-17 Despite accumulating mindfulness studies with this population, a paucity of literature exists as to the MBSR's effects in those with cancer-related pain. A single MBSR study reported significant improvement in pain intensity pre-post in breast cancer patients, ${ }^{18}$ however no published literature exists as to MBSR effects on pain catastrophizing, pain-related anxiety, or mental adjustment in those with cancer pain.

The aims of the present pre-post-intervention study were to address the gap in the literature byinvestigating the effects of a mindfulness intervention on pain catastrophizing, pain anxiety, and mental adjustment to cancer in women diagnosed with breast cancer. Data on pain intensity ratings also were collected for comprehensiveness of the pain experience. Examining intervention effects for pain catastrophizing and anxiety were exploratory, hence no hypotheses were made. Based on 
previous studies, it was hypothesized that scores for mental adjustment to cancer and pain intensity would improve significantly following the intervention.

\section{Materials and Methods}

\section{Design}

A one group pre-test/post-test design was used to assess changes in pain catastrophizing, pain-related anxiety, mental adjustment to cancer, and pain intensity before and after an eightweek MBSR intervention in women with breast cancer suffering with chronic cancer pain.

\section{Participants and procedures}

Following Human Subjects Approval, participants were recruited through medical facilities by fliers, media advertisements, and referrals from physicians and the American Cancer Society in a southwestern community of approximately 200,000 residents. The sample consisted of 41 participants with 31 women in stage II (76\%) and 10 (24\%) in stage III. The majority were Caucasian (96\%), Protestant (91\%), married (88\%), with a modal family income between $\$ 22,500$ and $\$ 67,110$ (82\%). Most were educated beyond high school (79\%), 14 women were working part-time (34\%), and seven women (17\%) indicated a family history of cancer. Cancer pain was confirmed by the attending oncologist in order to participate. Time from diagnosis ranged from 13-months to three years at the time of the intervention.

Exclusion criteria included: no cancer associated pain or chronic pain status, current primary sites other than breast, less than 18 years of age, and psychopathology. While not a criteria for exclusion, none of the women expressed consistent practice of formal meditation, the body scan or of hatha yoga. Individuals displaying psychopathology (i.e., psychotic symptoms or suicidal ideation) were referred to appropriate psychological services. Participants maintained their standard medical care during the program.

\section{Intervention}

The operational definition used for mindfulness meditation was the process of regulating attention so as to bring a quality of non-elaborative awareness to one's current experience as well as relating to one's experience with an orientation of curiosity, experiential openness, and acceptance. 14 The MBSR program 13 was held in a counseling center for 1.5 hours/week over eight-weeks. Participants were trained in the mindfulness practices of: body scan, sitting meditation, and hatha yoga. The body scan, as defined by Kabat-Zinn, is a gradual sweeping of attention throughout the entire body with non-critical acceptance of sensations or feelings, and suggestions of breath awareness and relaxation. 13 Sitting meditation, as discussed by Kabat-Zinn, is the practice of mindful attention of the breath with nonjudgmental awareness of distractions and returning to the breath focus. ${ }^{13}$ Hatha yoga, as explained by Kabat-Zinn with specific instructions, is the practice of simple stretches and postures to develop mindful movement of the body or meditation in motion. 13 The women received formal guidance and direction as to daily homework practice; compact discs were provided for personal use to facilitate daily homework of techniques learned in the weekly sessions. Outcome measures were completed before and following the intervention program.

\section{Outcome measures}

Four standardized instruments were administered before pre and after post the eight-week MBSR intervention to evaluate intervention effectiveness on the following dependent variables.

\section{Pain catastrophizing}

The Pain Catastrophizing Scale (PCS) was used in this study. 5 This 13 -item scale contains a 0 (not at all) to 4 (all the time) Likert- scale with rumination, magnification, and helplessness subscales. PC is considered to have a continuous distribution in the population without a clear cut-off level that distinguishes between high or low catastrophizers. ${ }^{8}$ Here, a total catastrophizing score was calculated from the sum of all items with higher scores indicating greater levels of catastrophizing. Good psychometric properties of construct validity and reliability in both clinical and non-clinical samples have been demonstrated. 5

\section{Pain anxiety}

The Pain Anxiety Symptoms Scale-20 (PASS-20) was used to measure pain-related anxiety. 18

Items are rated on a 6-point Likert scale from 0 (never) to 5 (always), and scores range between 0-100 with higher scores indicating greater levels of anxiety. The reliability and validity of the PASS-20 are well established. ${ }^{19}$

\begin{tabular}{|c|c|c|c|c|c|}
\hline \multirow[b]{2}{*}{ Variable } & \multicolumn{2}{|c|}{ Preintervention } & \multicolumn{2}{|c|}{ Postintervention } & \multirow[b]{2}{*}{$t$} \\
\hline & $M$ & SD & $M$ & SD & \\
\hline Pain Catastrophizing (PCS) & 43.13 & 12.04 & 40.78 & 9.06 & $2.88^{*}$ \\
\hline Pain Anxiety (PASS-20) & 64.07 & 8.32 & 59.71 & 11.01 & $4.69^{* *}$ \\
\hline Anxious Preoccupation (MAC) & 28.63 & 7.39 & 24.73 & 7.00 & $4.97^{* *}$ \\
\hline Hopeless-Helplessness (MAC) & 17.43 & 10.01 & 14.88 & 9.93 & $2.98^{*}$ \\
\hline Pain Rating (MPQ-SF) & 34.94 & 8.23 & 32.00 & 8.66 & $3.01^{*}$ \\
\hline
\end{tabular}

\section{Mental adjustment to cancer}

The Mental Adjustment to Cancer Scale (MAC) was used to assess psychological adjustment to cancer. ${ }^{11}$ The MAC scale is a 40 -item questionnaire using a four-point Likert scale, which measures five mental and behavioral attitudinal coping styles: Fighting Spirit (FS), Helpless-Hopelessness (HH), Anxious Preoccupation (AP), Stoicism-Fatalism (FA), and Avoidance-Denial. Items are rated on a scale ranging from definitely does not apply to me (1) to definitely applies to me (4). Acceptable psychometric properties of the MAC have been reported and replicated. ${ }^{11}$ The avoidance subscale was not included because it consists of only one item.

\section{Pain intensity}

The pain rating index (PRI) of the McGill Pain Questionnaire, short form (MPQ-SF) was used to measure pain intensity, which has been used with cancer populations. ${ }^{20}$ The pain rating index has two dimensions: the sensory dimension consisting of 11 items; and, the affective dimension consisting of 4 items. The pain level was determined by adding the ratings of 15 items in the PRI (range 0 to 45) with higher scores indicating more severe pain. The MPQ-SF has demonstrated construct validity as well as internal reliability and test-retest reliability of 0.88 to $0.96 .{ }^{20}$

\section{Results}

Paired t-test analyses were conducted using an SPSS software program to assess pre-and-post changes for each outcome variable. (Table 1, Figure 1).

\section{Pain catastrophizing}

Results for pain catastrophizing showed that scores dropped significantly from before the intervention $(\mathrm{M}=43.13, \mathrm{SD}=12.04)$ to the end of the eight-week MBSR program $(\mathrm{M}=40.78, \mathrm{SD}=9.06)(\mathrm{t}=2.88, \mathrm{P}<0.05)$. Thus, the women in this sample reported a significant reduction in being negatively preoccupied with their cancer-related pain.
Table 1. Means and standard deviations dependent variables pre-post intervention. 


\section{Pain anxiety}

Analyses of pre-post scores for pain-related anxiety revealed positive and significant changes from baseline $(\mathrm{M}=64.07, \mathrm{SD}=8.32)$ to the end of the MBSR intervention ( $M=59.71$, $\mathrm{SD}=11.01),(\mathrm{t}=4.69, \mathrm{P}<0.01)$. These findings indicate that this group of women with breast cancer experienced significant improvement pre-to-post-intervention for anxiety specifically associated with pain.

\section{Mental adjustment to cancer}

Two of the coping responses to cancer, anxious preoccupation (AP) and helpless-hopelessness $(\mathrm{HH})$, showed significant changes in scores before and following the MBSR program. Specifically, baseline scores on the AP subscale $(\mathrm{M}=28.63, \mathrm{SD}=7.39)$ decreased significantly by the end of the 8-week period ( $\mathrm{M}=$ 24.73, $\mathrm{SD}=7.00),(\mathrm{t}=4.97, \mathrm{P}<0.01)$. Also, scores on the HH subscale decreased significantly pre-intervention $(\mathrm{M}=17.43, \mathrm{SD}=10.01)$ to postintervention $(\mathrm{M}=14.88, \mathrm{SD}=9.93),(\mathrm{t}=2.98$, $\mathrm{P}<0.05)$. Thus, as predicted, beneficial significant improvements in mental adjustment occurred following the MBSR program.

\section{Pain intensity}

It was predicted that pain intensity would significantly improve as indicated by a reduction in pre-to-post scores for pain intensity ratings as measured by the MPQ-SF. Results indicated that, indeed, scores prior to the intervention $(\mathrm{M}=34.94, \mathrm{SD}=8.23)$ decreased significantly eight-weeks later after the end of the MBSR intervention ( $\mathrm{M}=32.00, \quad \mathrm{SD}=8.66)$ $(\mathrm{t}=3.01, \mathrm{P}<0.05)$. The hypothesis that pain ratings would decrease significantly after the MBSR was supported.

\section{Discussion}

This was the first known MBSR study to investigate intervention effects on pain catastrophizing in women diagnosed with stage II and stage III breast cancer, who were experiencing cancer-related pain. Pain catastrophizing is an important risk factor for chronic pain and disability; additionally, it is central to fearavoidance model because it is viewed as the cognitive route through which the fear of pain develops. 8,10 This negative appraisal is important because it contributes to the variance in patients' subjective reporting of their pain severity. ${ }^{8}$ In this study, participants' pain catastrophizing scores dropped significantly following completion of the MBSR intervention. Pain-related anxiety also was explored for the first time in a mindfulness-based intervention study in cancer participants; results showed significant improvement in pre-post scores.

\section{Pre-Post Changes}

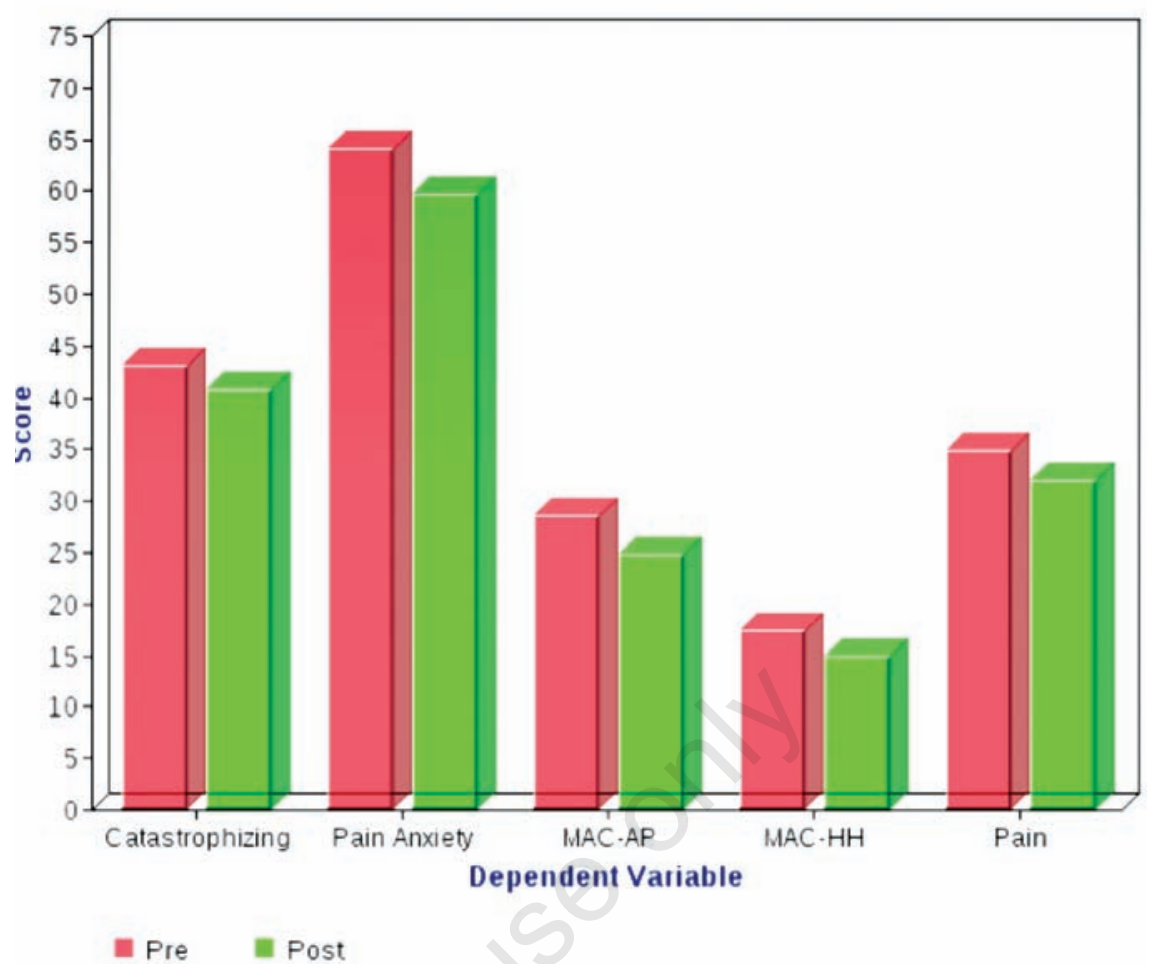

Figure 1. Pre-post changes of dependent variables.

Previous findings documented significant improvements in state anxiety levels among individuals with cancer following an MBSR program, 16 yet it was unknown if similar effects would be found for pain-related anxiety. Mental adjustment to cancer and pain ratings changed significantly and positively in this study. When conducting the MBSR program, limitations must be acknowledged. First, the program includes practices (e.g., body scan, yoga) in addition to the traditional meditation training; therefore, the inability to ascertain whether intervention effects were due to one practice or a combination thereof is an inherent limitation. Next, this was a pre-post design lasting for eight weeks. It is recommended that future studies examine longer term effects as well as include a depression measure since many cancer patients suffer from mood changes, which this study did not address. Lastly, it is recommended that future studies include a comparison group to assess between group differences. In conclusion, this was the first MBSR-cancer exploration of pain factors in patients with cancer pain. These findings need to be interpreted cautiously, for replication and future investigations need to be conducted in this line of research. The results of this study, however, provide data in the neglected area regarding cancer patients with pain.

\section{References}

1. Bishop SR, Warr D. Coping, catastrophizing and chronic pain in breast cancer. $\mathrm{J}$ Behav Med 2003;26:265-81.

2. Melzack R, Wall PD. Pain mechanisms: a new theory. Science 1965;150:971-9.

3. Raphael J, Hester J, Ahmedzai S et al. Cancer pain: Part 1. Pathophysiology; Oncological, pharmacological, and psychological treatments: A perspective from the British Pain Society endorsed by the UK Association of Palliative Medicine and the Royal College of General Practitioners. Pain Med 2010;11:742-764.

4. Foley KM. Advances in cancer pain management in 2005. Gyn Oncol 2005;99:S126.

5. Sullivan MJ, Bishop SC, Pivik J. The Pain Catastrophizing Scale: Development andd validation. Psychol Assess 1995;7:524-32.

6. Greer S, Moorey S, Watson M. Adjustment to cancer: the Mental Adjustment to Cancer (MAC) scale vs clinical ratings. J Psychosomat Rsh 1989;33:373-7.

7. Asmundson GJ, Taylor S. PTSD and chronic pain: cognitive-behavioral perspectives and practical implications. Causality: psychological knowledge and evidence in court In: G. Young, A.W. Kane and K. Nicholson, Editors Springer, New York: 
2006. pp. 225-241.

8. Sullivan MJ, Thorn B, Haythornthwaite J et al. Theoretical perspectives on the relation between catastrophizing and pain. Clin J Pain 2001;17:52-64.

9. Schroevers M, Kraaij V, Garnefski N. How do cancer patients manage unattainable personal goals and regulate their emotions? Br J Hlth Psychol 2008;13:551-62.

10. Vlaeyen JW Linton SJ. Fear-avoidance and its consequences in chronic musculoskeletal pain: a state of the art. Pain 2000;85:317-32.

11. Watson M, Greer S, Young $\mathrm{J}$ et al. Development of a questionnaire measure of adjustment to cancer: the MAC scale. Psychol Med 1988;18:203-9.

12. Tacón A, Caldera Y, Ronaghan C. Mindfulness-based stress reduction in women with breast cancer. Fam Sys Hith 2004;22:193-203.

13. Kabat-Zinn J. Full catastrophe living: Using the wisdom of your body and mind to face stress, pain, and illness. Dell; New York: 1990.

14. Bishop S, Lau M, Shapiro S et al. Mindfulness: A Proposed Operational Definition. Clin Psychol Sci Prac 2004; 11: 230-41.

15. Carlson L, Garland S. Impact of mindfulness-based stress reduction on sleep, mood, stress and fatigue symptoms in cancer outpatients. Int J Behav Med 2005;12: 278-85.

16. Speca M, Carlson L, Goodey E, Angen M. A randomized, wait-list controlled clinical trial: The effect of a mindfulness meditation based stress reduction program on mood and symptoms of stress in cancer outpatients. Psychosomat Med 2000:62: 613-22.

17. Tacón A, Caldera Y, Ronaghan C. Mindfulness, psychosocial factors and breast cancer. J Ca Pain Symptom Palliat 2005;1:45-54

18. Tacón, A. Mindfulness effects on symptoms of distress in women with cancer. $\mathrm{J}$ Cancer Pain Symptom Palliat 2006;2:1722.

19. McCracken LM, Dhingra, L. A short version of the Pain Anxiety Symptoms Scale (PASS-20): preliminary development and validity. Pain Rsh Manag 2002;7:45-50.

20. Melzak R. The short-form McGill Pain Questionnaire. Pain 1987;30:191-7. 